\title{
Future application of deubiquitylating enzymes for rapid and efficient cellular reprogramming
}

\begin{abstract}
In stem cell pluripotency and differentiation, ubiquitylation and deubiquitylation modifications of stem cell core transcription proteinsis of high significance, as it influences stem cell fate determination. Two key enzymes i.e. an E3 ubiquitin ligase enzyme; known for tagging ubiquitin molecules to the target proteins for degradation, and the deubiquitylating enzyme (DUB), that counteracts the proteolysis, have to be well balanced for cellular homeostasis. Interestingly, the notion of DUBs enhancing the stability and half-life of stem cell core transcription factors has presented a new vision about significance of applying DUBs to control stem cell fate determination and cellular reprogramming. In this review, we propose the applications of DUBs along with stem cells core transcription factors i.e. Oct4, Sox2, Klf4, c-Myc, Nanog and Lin28 for an efficient generation of protein induced pluripotent stem cells.
\end{abstract}

Keywords: stem cell fate determination, post-translational modifications, proteininduced pluripotent stem cells, transcriptional regulation, cellular reprogramming, ipsc generation, protein half-life
Volume 2 Issue 6 - 2017

\author{
Saba Haq,' Suresh Ramakrishna ${ }^{2,3}$ \\ 'Department of Life Science, College of Natural Sciences, \\ Hanyang University, Seoul, South Korea \\ ${ }^{2}$ Graduate School of Biomedical Science and Engineering, \\ Hanyang University, Seoul, Korea \\ ${ }^{3}$ College of Medicine, Hanyang University, Seoul, South Korea
}

Correspondence: Suresh Ramakrishna, Assistant Professor, Department of Biomedical Science, Graduate School of Biomedical Science \& Engineering, Hanyang University, South Korea, Tel +82222202424, Fax +82222202422,

Email suri28@hanyang.ac.kr, suresh.ramakris@gmail.com

Received: April 05, 2017 | Published: June 08, 2017
Abbreviations: DUB, deubiquitylating enzyme; ESCs, embryonic stem cells; ICM, inner cell mass; PTM, post-translational modification; iPSCs, induced pluripotent stem cells; USP, ubiquitin-specific processing protease; $\mathrm{UCH}$, ubiquitin carboxy-terminal hydrolase; OUT, otu-domain ubiquitin aldehyde binding protein; JAMM, jad1/pad/mpn-domain-containing metalloenzyme; MCPIP, monocyte chemotactic protein-induced protease; PSC, pluripotent stem cell; CNS, central nervous system; CPP, cell penetrating peptide; piPSCs, protein-induced pluripotentstem cells; WWP2, ww domain-containing protein 2; UPS, ubiquitin proteasome system; MEFs, mouse embryonic fibroblasts; FBXW8, f-box/wd repeat-containing protein 8

\section{Introduction}

Embryonic stem cells (ESCs) are derived from the inner cell mass (ICM) of the blastocyst and possess the unique ability for unlimited self-renewal. ESCs can differentiate into three embryonic germ layers

a) ectoderm - nerve and skin,

b) mesoderm - bone, muscle, and blood

c) endoderm - lung tissues and gut

During cell division, each stem cell can either remain as stem cell or differentiate into a particular cell type. Several reports indicate the role of transcriptional factors such as c-Myc, Sox2, Oct4, Klf4, Lin28 and Nanog, in controlling the regulation of cellular reprogramming, stem cell pluripotency and differentiation. ${ }^{1,2}$ In addition, posttranslational modifications (PTMs) play a central part in directing multiple cellular processes through protein regulation.

PTMs such as sumoylation and phosphorylation are involved in the regulation of pluripotency-related transcriptional pathways. ${ }^{1,2}$ PTMs also include the key regulatory cellular mechanism of ubiquitylation, which reverses upon deubiquitylation by DUBs. However, the information about the roles of ubiquitylation and deubiquitylation processes on pluripotency-associated transcriptional networks of ESCs is limited. This review article demonstrates the significance of DUBs in regulating various proteins involved in the process of cellular reprogramming such as induced pluripotent stem cell (iPSC) generation and its further applications.

\section{Ubiquitin-proteasome pathway}

The process of ubiquitylation includes conjugation of ubiquitin molecule to a protein substrate, thereby regulating its stability and function. For the conjugation of ubiquitin moiety to a target protein, the sequential activity of three classes of enzymes i.e. E1 (ubiquitin activating enzymes), E2 (ubiquitin conjugating enzymes), and E3 (ubiquitin ligases) is necessary ${ }^{3-5}$ (Figure 1). Ubiquitin is a small and highly conserved protein which can covalently attach to protein substrates as a single unit (monoubiquitin), multiple units (multimonoubiquitins) or in the form of a polyubiquitin chain. ${ }^{6,7}$ During polyubiquitylation, any of the seven lysine $(\mathrm{K})$ residues (K6, K11, $\mathrm{K} 27, \mathrm{~K} 29, \mathrm{~K} 33$, K48, and K63) of ubiquitin can form linkages to others resulting into a sizeable chain comprising of sequentially linked ubiquitin molecules. Polyubiquitin chains formed by K-48 and K-63 linkage have been well understood and are widely reported in literature. K-48 linked chains directs the target protein for proteolysis via $26 \mathrm{~S}$ proteasome, whereas K-63 linked polyubiquitin chains are involved in regulation of protein activity and signal transduction events. ${ }^{7}$ Thus, ubiquitylation is a significant regulatory mechanism that is involved in various cellular processes such as apoptosis, oncogenesis, immune response, transcriptional DNA repair regulation, cell cycle control, embryonic development and intracellular signaling pathways.? 


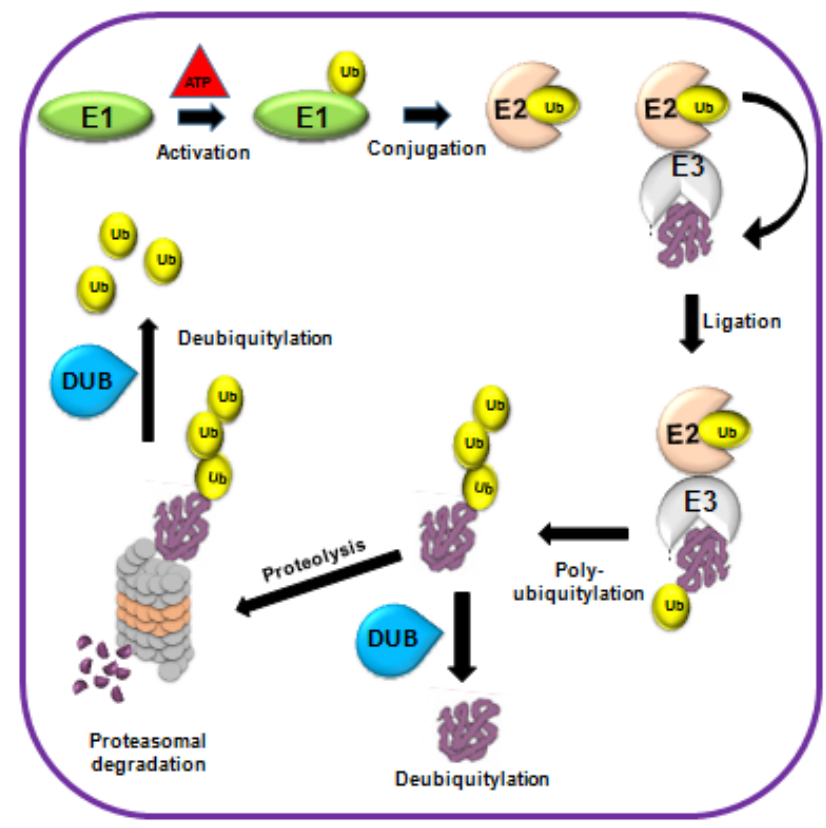

Figure I The ubiquitin proteasomal pathway. The sequential activity of EI (ubiquitin activating enzymes), E2 (ubiquitin conjugating enzymes), and E3 (ubiquitin ligases) ligates ubiquitin moiety to the protein substrate. Protein substrates attached with lysine 48 linked polyubiquitylation are targeted to the $26 \mathrm{~S}$ proteasome for target protein proteolysis. Ubiquitin molecules are recycled through the activity of DUBs.

\section{Deubiquitylation}

DUBs are proteases which cleave ubiquitin moiety from ubiquitinconjugated protein substrates as well as ubiquitin-ubiquitin covalent links. DUBs counteract ubiquitylation and consequently prevent proteasome-dependent protein degradation. Thus, deubiquitylation is an important event in maintaining ubiquitin homeostasis (Figure 1). DUBs are involved in: recycling of ubiquitin molecules from a previous ubiquitylation event, removal of nonessential ubiquitin molecules, and editing and rearranging the ubiquitylation process to modify the target protein accordingly. ${ }^{8}$

Approximately 100 different kinds of DUBs have been found in the human genome which has been classified into six broad families:

i. Ubiquitin-specific processing protease (USP/UBP),

ii. Ubiquitin carboxy-terminal hydrolase (UCH),

iii. Otu-domain ubiquitin aldehyde binding protein (OTU),

iv. Jad1/Pad/MPN-domain-containing metalloenzyme (JAMM),

v. Monocyte chemotactic protein-induced protease (MCPIP) and

vi. Ataxin-3/Josephin proteases.

Among these categories, the USP family is the largest and consists of more than 50 members. ${ }^{8,9}$ DUBs have been reported to recycle ubiquitin molecules, cleave polyubiquitin chains, process ubiquitin precursors and reverse ubiquitin conjugation. ${ }^{8}$ Similar to ubiquitylation, DUBs are also involved in the regulation of numerous cellular activities such as proteasome-dependent and lysosome-dependent proteolysis, DNA repair, gene expression, chromosome segregation, kinase activation, cell cycle progression, apoptosis, localization, spermatogenesis, and degradation of signaling intermediates. ${ }^{8,10,11}$
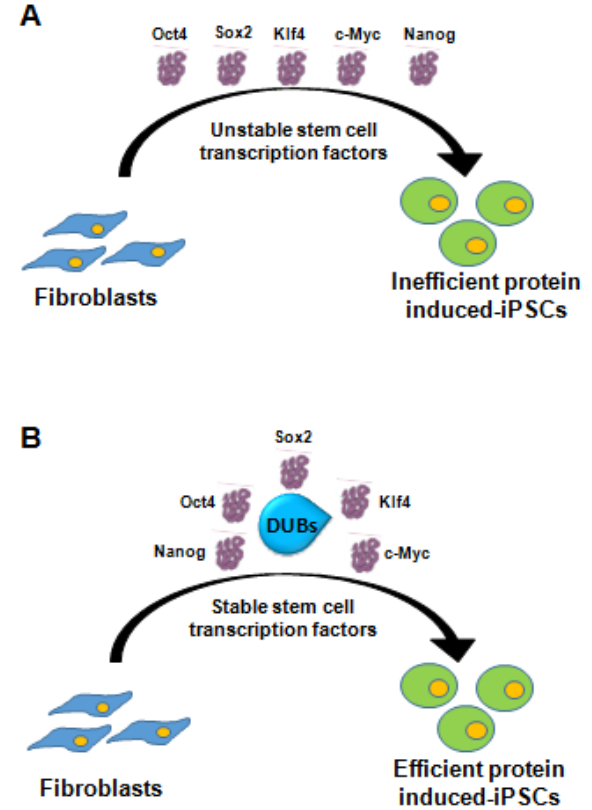

Figure 2 iPSC generation in the presence or absence of DUBs. (A) Figure demonstrates the mechanism of iPSC generation from fibroblasts in the absence of DUBs thereby leading towards inefficient production. (B) Figure shows diagrammatical representation of iPSC generation in the presence of DUBs leading towards stabilized stem cell core transcription factors and subsequent efficient iPSC production.

\section{Application of DUBs for rapid and efficient iPSC ge- neration}

Identification of pluripotent stem cell (PSC)-specific genes is crucial for comprehending the mechanism of stemness. Previous studies have identified several key factors such as POU5F1 (Oct3/4, Oct4), Sox2, and Nanog for controlling the pluripotent state. ${ }^{12-15}$ Nanog and Oct4 were discovered due to their confined expression in ESCs; Sox2, on the other hand, was found to be expressed in other multi-potential cells and precursor cells of developing central nervous system (CNS) ${ }^{16}$ Nanog, Oct4 and Sox 2 transcription factors bind to DNA and thus, this triumvirate controls the subsequent gene expression. ${ }^{17,18}$ Yamanaka factors (Sox2, Oct3/4, c-Myc, Klf4) exhibit high expression in ESCs, resulting into pluripotency induction in mouse and human somatic cells, thereby, demonstrating a vital role in regulation of the signaling network involved in ESCs fate choice. ${ }^{19}$

iPSCs generation evades the political and ethical concerns of using oocytes and embryos. The possibility of iPSCs generation from patients confers it as a safe technique as compared to ESCs and present unprecedented opportunities for research and applications in clinical settings. However, various limitations need to be addressed in order to design reliable iPSCs generation methods for clinical applications. Currently existing methods for generating iPSCs include lentiviral, episomal, mini circle, send virus and direct delivery of proteins. Primary concerns with retroviral or lentiviral vectors include the transgene integration followed by mutations in host DNA. Another concern during iPSC generation is the reactivation of transcriptionally silent proviruses that could promote carcinogenesis. ${ }^{20,21}$ In contrast, transiently expressing vectors e.g. Adenoviral or episomal vectors cause a very low frequency of iPSC generation. ${ }^{23,24}$ Due to viral methodologies based drawbacks, non-viral based methods have gained attention. To address the safety concerns arising from harboring 
integrated exogenous sequences in the genome of the cell, numerous genetic methods have been designed to reduce the risk. ${ }^{23}$ However, use of genetic materials in such methods, raises the probability for unexpected genetic alterations by the exogenous sequences in the target genome. Among these, a novel strategy of human iPSCs generation by direct delivery of reprogramming related proteins has been designed.

The process of proteins cellular delivery includes their conjugation with a short cell penetrating peptide (CPP). A poly-arginine (11R) CPP conjugated to the carboxyl terminal of reprogramming factors, i.e. Klf4, Oct4, Sox2, and c-Myc was introduced into the cells. ${ }^{25}$ Such reprogrammed cells with transduced proteins are called proteininduced pluripotent stem cells (piPSCs). Later an advanced method for iPSC generation was introduced by utilizing direct delivery of reprogramming proteins without any chemical treatment. ${ }^{26}$ Protein-based iPSCs have greater efficacy of generating functional dopamine neurons as compared to virus-based iPSC. Moreover protein-based iPSCs have been found to rescue motor defects in the Parkinson disease model..$^{27}$ The protein based transduction method offers a substantially simpler strategy as compared to the genetic based methods that demand time-consuming sequential selection of integration-free iPSCs. However, piPSC based methodology takes approximately double time in contrast with viral transduction-based iPSC generation. Additionally, unstable proteins with low half-lives followed by its lower efficiency in generating piPSCs are the few hurdles that have to be overcome for successful protein based cellular reprogramming.

The half-life of stem cell core transcription factors is directly proportional to the effectiveness of pIPSCs generation. Interestingly, it has been confirmed that all the Yamanaka transcription factors undergo ubiquitylation through $26 \mathrm{~S}$ proteasome and have relatively low halflives. For example, Oct4 protein degrades at a rapid pace and exhibits a short half-life of about 90 minutes. ${ }^{28}$ In ESCs, Oct4 ubiquitylation events are promoted by WW domain-containing protein 2 (WWP2), which is also involved in negative regulation of Oct4 transcriptional activity. ${ }^{29}$ Similarly, another stem cell core transcriptional factor i.e. Klf4 also degrades rapidly and has a short half-life of about 120 minutes. Moreover, proteasome inhibitor MG132-treatment extends half-life of Klf4 protein by partial refractory to its protein degradation. Increased amount of ubiquitin bound Klf4 proteins in proliferating cells in contrast to the serum-starved cells, demonstrate the significance of ubiquitylation in serum-mediated degradation. ${ }^{30}$ Klf4 levels enhance drastically after MG132 treatment demonstrating that it undergoes proteasomal degradation. ${ }^{31} \mathrm{c}-\mathrm{Myc}$, being a highly unstable protein, has a half-life of only $20-30$ minutes. ${ }^{32}$ Nanog protein exhibits a relatively short half-life of approximately 120 minutes in human ESCs. ${ }^{33}$ It is generally known that ubiquitin proteasome system (UPS) degrades numerous short-lived regulatory proteins in vivo. ${ }^{34}$ Upon pretreatment with MG132, Nanog protein stability and its halflife extends in human ESCs. Moreover, a PEST motif sequence (rich in glutamine, proline, threonine and serine) ranging from $47^{\text {th }}$ to $72^{\text {nd }}$ amino acid in N-terminal of Nanog has been found to be involved in protein proteolysis. Deletion of PEST motif leads to decrease in ubiquitylation levels of Nanog, thereby causing stabilization of Nanog. ${ }^{33}$ However, further investigation of stabilized Nanog protein without PEST motif sequences in iPSC generation has to be carried out.

DUBs have been found to play a significant part in regulating stemness. For instance; knockdown of USP34 augments the mammosphere-forming ability concomitant with the significant upregulation of Oct4, Nanog and Sox 2 mRNA levels in mammary epithelial cells in vitro. ${ }^{35}$ USP44 confers a high expression in PSCs as well as germinal cells/organs; however the expression levels are repressed upon cellular differentiation and also in somatic tissues. Epigenetically, the transcription of USP44 correlates with the DNA methylation of a $\mathrm{CpG}$ island near its key promoter area. The study suggests that the expression profile of USP44 in PSCs is controlled by complex machinery that may involve interaction of pluripotent core genes (POU5F1, Sox 2 and Nanog) with other epigenetic factors. ${ }^{36}$ The role of USP44 in PSCs needs to be further characterized. TRIM32 is a well-known ubiquitin ligase that ubiquitylates c-Myc and Oct 4 proteins. The absence of TRIM32 enhances the efficacy of reprogramming from mouse embryonic fibroblasts (MEFs) into iPSCs. ${ }^{37}$ USP28, USP36 and USP37 have been reported to deubiquitylate and stabilize c-Myc. ${ }^{38-40}$ Klf4 protein has a high turn-over rate and it is a target for proteasome-mediated proteolysis by pVHL however DUB for Klf4 is not well defined. ${ }^{31}$ USP21 deubiquitylates and stabilizes Nanog and reverses the F-box/WD repeat-containing protein 8 (FBXW8)mediated Lys48-linked polyubiquitylation of Nanog. . $^{41,42}$ Thus, USP2 1 might have a significant role in maintaining the pluripotency of iPSCs and ESCs, iPSCs generation and offers novel insights for therapeutic interventions. ${ }^{42}$

The aforementioned examples vividly illustrate that Yamanaka factor proteins are undergoing ubiquitylation and subsequent proteolysis. In order to extend the half-life and stability of a particular protein, a suitable strategy is to identify potential DUB candidates that could reverse their ubiquitylation and protein degradation. Thus, screening for potential DUBs, that could regulate the protein levels of core stem cell transcription factors might offer novel applications. For instance, the combined delivery of potential DUBs candidate regulating protein degradation of stem cell transcription factors coupled with the Yamanaka factors during cellular reprogramming is a promising new approach. Utilizing this paradigm of reciprocal posttranslational regulatory modulations by DUBs in stem cell networks during iPSCs generation might considerably improve the efficiency of cellular reprogramming and pave way for further applications in the related field.

Alternatively, identification of critical lysine residues in core stem cell transcriptional factors for the purpose of protein stabilization is a potential strategy for improving the efficiency of cellular reprogramming. In-depth bioinformatics analysis has predicted potential ubiquitylation related several lysine residues in core stem cell transcriptional factors. For an instance, a link between lysine site $(\mathrm{K})$ at $232^{\text {nd }}$ position with ubiquitylation has been established in Klf4 protein. Mutant lysine 232 has been reported to exhibit higher stability and longer half-life as compared to its non-mutant counterpart. ${ }^{43}$ Similarly, mutation of K43 lysine residue alone or in combination with K23R has been found to reduce the ubiquitylation levels of Klf4. ${ }^{31}$ We recommend further investigations in the similar direction for improving the stability of Yamanaka factors. Thus, a novel strategy of replacing predicted ubiquitylation mediating lysine sites of core stem cell transcriptional factors might contribute to improving the stability of such proteins thereby, leading towards efficient protein-induced iPSCs generation. pIPSC is a promising technique for the production of patient-specific iPSCs however few drawbacks like complications in protein purification and less efficiency have to be addressed. Taken together, purification as well as 
expression of modified core transcriptional factor proteins with longer half-life might contribute to better protein-induced iPSCs generation efficiency.

\section{Conclusion and future prospects}

Stem cell fate results from a delicate check and balance between ubiquitylation and deubiquitylation regulatory events. Identification of DUBs candidates which could reverse the degradation of core stem cell transcription factors would be essential to comprehend the molecular pathways influencingthe fate determination of ESCs. In addition, screening of particular DUBs for factors associated with the direct conversion of fibroblasts to functional neurons ${ }^{44}$ endothelial cells, ${ }^{45}$ astrocytes ${ }^{46}$ hepatocytes ${ }^{47}$ etc., might confer noteworthy implications on iPSC related research studies. In conclusion, extensive mapping of the crosstalk between ubiquitylation and deubiquitylation events in the context of the regulation of Yamanaka factors is of high significance. Two approaches mentioned in our review i.e. identifying DUBs for stem cell core transcriptional factors and mapping ubiquitylation sites on such Yamanaka factors might lead towards stable and efficient piPSC production. After careful consideration of short half-lives of c-Myc (20-30minutes), Oct4 (90minutes), Nanog and Klf4 proteins (120minutes),we anticipate that there is dire need to identify critical lysine residue of these proteins to extend their short half-lives. Thus, maintenance of stable and efficient Yamanaka factors and their use for protein-induced iPSCs generation might have boundless outcomes in context to cellular reprogramming

We further recommend that the alternate unexplored concept of "Dubbing DUBs" i.e. deubiquitylating enzymes itself undergoing self or trans-deubiquitylation event should also be considered with reference to efficient iPSC generation. We speculate that any DUB involved in regulation of iPSC generation might also undergo ubiquitylation. Thus, identification of additional novel DUBs which can stabilize those DUBs which are already known to regulate iPSC generation is required. ${ }^{48} \mathrm{We}$ anticipate that corresponding research will not only lead towards exciting novel avenues for future investigation but also initiate DUB-targeted treatment approaches. However, detailed research still remains to be done in order to corroborate and expand the concept of DUBs-mediated iPSC generation for future clinical applications.

\section{Acknowledgements}

We would like to thank all of Suri's laboratory members for their helpful discussions. This study was supported by a grant of the National Research Foundation of Korea (2015R1C1A1A01054482).

\section{Conflict of interest}

The author declares no conflict of interest.

\section{References}

1. Cai N, Li M, Qu J, et al. Post-translational modulation of pluripotency. J Mol Cell Biol. 20124(4):262-265.

2. Ramakrishna S, Kim KS, Baek KH. Posttranslational modifications of defined embryonic reprogramming transcription factors. Cellular reprogram. 2014;16(2):108-120.

3. Hershko A, Ciechanover A, Rose IA. Identification of the active amino acid residue of the polypeptide of ATP-dependent protein breakdown. $J$ Biol Chem. 1981;256(4):1525-1528.
4. Hershko A, Heller H, Elias S, et al. Components of ubiquitin-protein ligase system. Resolution, affinity purification, and role in protein breakdown. J Biol Chem. 1983;258(13):8206-8214.

5. Hershko A, Ciechanover A. The ubiquitin system. Annu Rev Biochem. 1998;67:425-479.

6. Fang S, Weissman AM. A field guide to ubiquitylation. Cell Mol Life Sci. 2004;61(13):1546-1561.

7. Akutsu M, Dikic I, Bremm A. Ubiquitin chain diversity at a glance Journal of Cell Science. 2016;129:875-880.

8. Turcu FER, Ventii KH, Wilkinson KD. Regulation and Cellular Roles of Ubiquitin-specific Deubiquitinating Enzymes. Annu Rev Biochem. 2009;78:363-397.

9. Amerik AY, Hochstrasser M. Mechanism and function of deubiquitinating enzymes. Biochim Biophys Acta. 2004;1695(1-3):189-207.

10. Ramakrishna S, Suresh B, Baek KH. The role of deubiquitinating enzymes in apoptosis. Cell Mol Life Sci. 2011;68(1):15-26.

11. Ramakrishna S, Suresh B, Baek KH. Biological functions of hyaluronan and cytokine-inducible deubiquitinating enzymes. Biochimica et Biophysica Acta (BBA)-Reviews on Cancer. 2015;1855(1):83-91.

12. Masui S, Nakatake Y, Toyooka Y, et al. Pluripotency governed by Sox2 via regulation of Oct $3 / 4$ expression in mouse embryonic stem cells. Nat Cell Biol. 2007;9:625-635.

13. Mitsui K, Tokuzawa Y, Itoh H, et al. The Homeoprotein Nanog Is Required for Maintenance of Pluripotency in Mouse Epiblast and ES Cells. Cell. 2003;113(5):631-642.

14. Chambers I, Colby D, Robertson M, et al. Functional Expression Cloning of Nanog, a Pluripotency Sustaining Factor in Embryonic Stem Cells. Cell. 2003;113(5):643-655.

15. Nichols J, Zevnik B, Anastassiadis K, et al. Formation of Pluripotent Stem Cells in the Mammalian Embryo Depends on the POU Transcription Factor Oct4. Cell. 1998;95(3):379-391.

16. Fong H, Hohenstein KA, Donovan PJ. Regulation of Self-Renewal and Pluripotency by Sox2 in Human Embryonic Stem Cells. Stem cells. 2008;26(8):1931-1938.

17. Young Richard A. Control of the Embryonic Stem Cell State. Cell. 2011;144(6):940-954.

18. Chambers I, Tomlinson SR. The transcriptional foundation of pluripotency. Development (Cambridge, England). 2009;136(14):2311-2322.

19. Liu X, Huang J, Chen T, et al. Yamanaka factors critically regulate the developmental signaling network in mouse embryonic stem cells. Cell Res. 2008;18(12):1177-1189.

20. Hacein Bey Abina S, Von Kalle C, Schmidt M, et al. A Serious Adverse Event after Successful Gene Therapy for X-Linked Severe Combined Immunodeficiency. N Engl J Med. 2003;348(3):255-256.

21. Hacein-Bey-Abina S, Von Kalle C, Schmidt M, et al. LMO2 Associated Clonal T Cell Proliferation in Two Patients after Gene Therapy for SCID-X1. Science. 2003;302(5644):415-419.

22. Stadtfeld M, Nagaya M, Utikal J, et al. Induced pluripotent stem cells generated without viral integration. Science. 2008;322(5903):945-949.

23. Yamanaka S. A Fresh Look at iPS Cells. Cell. 2009;137(1):13-17.

24. Zhou W, Freed CR. Adenoviral Gene Delivery Can Reprogram Human Fibroblasts to Induced Pluripotent Stem Cells. Stem cells. 2009;27(1):2667-2674. 
25. Zhou H, Wu S, Joo JY, et al. Generation of Induced Pluripotent Stem Cells Using Recombinant Proteins. Cell Stem Cell. 2009;4(5):381-384.

26. Kim D, Kim CH, Moon JI, et al. Generation of Human Induced Pluripotent Stem Cells by Direct Delivery of Reprogramming Proteins. Cell stem cell. 2009;4(6):472-476.

27. Rhee YH, Ko JY, Chang MY, et al. Protein-based human iPS cells efficiently generate functional dopamine neurons and can treat a rat model of Parkinson disease. J Clin Invest. 2011;121(6):2326-2335.

28. Saxe JP, Tomilin A, Scholer HR, et al. Post-translational regulation of Oct4 transcriptional activity. PLoS One. 2009;4(2):e4467.

29. Xu HM, Liao B, Zhang QJ, et al. Wwp2, an E3 ubiquitin ligase that targets transcription factor Oct-4 for ubiquitination. J Biol Chem. 2004;279(22):23495-23503.

30. Chen ZY, Wang X, Zhou Y, et al. Destabilization of Kruppel-like factor 4 protein in response to serum stimulation involves the ubiquitin-proteasome pathway. Cancer Res. 2005;65(22):10394-10400.

31. Gamper AM, Qiao X, Kim J, et al. Regulation of KLF4 turnover reveals an unexpected tissue specific role of pVHL in tumorigenesis. Mol cell. 2012;45(2):233-243.

32. Hann SR, Eisenman RN. Proteins encoded by the human c-myc oncogene: differential expression in neoplastic cells. Mol Cell Biol. 1984;4(11):2486-2497.

33. Ramakrishna S, Suresh B, Lim KH, et al. PEST Motif Sequence Regulating Human NANOG for Proteasomal Degradation. Stem Cells Dev. 2011;20(9):1511-1519.

34. Reed SI. Ratchets and clocks: the cell cycle, ubiquitylation and protein turnover. Nat Rev Mol Cell Biol. 2003;4(11):855-864.

35. Oh E, Kim JY, Sung D, et al. Inhibition of ubiquitin-specific protease 34 (USP34) induces epithelial-mesenchymal transition and promotes stemness in mammary epithelial cells. Cell Signal. 2017;36:230-239.

36. Tropel P, Jung L, André C, et al. CpG island methylation correlates with the use of alternative promoter for USP44 gene expression in human pluripotent stem cells and testis. Stem Cells Dev. 2017;26(15):11001110 .
37. Bahnassawy La, Perumal TM, Gonzalez Cano L, et al. TRIM32 modulates pluripotency entry and exit by directly regulating Oct4 stability. $\mathrm{Sci}$ Rep. 2015;5:13456.

38. Sun XX, He X, Yin L, et al. The nucleolar ubiquitin-specific protease USP36 deubiquitinates and stabilizes c-Myc. Natl Acad Sci U S A. 2015;112(12):3734-3739.

39. Popov N, Wanzel M, Madiredjo M, et al. The ubiquitin-specific protease USP28 is required for MYC stability. Nat Cell Biol. 2007;9(7):765-774.

40. Pan J, Deng Q, Jiang C, et al. USP37 directly deubiquitinates and stabilizes c-Myc in lung cancer. Oncogene. 2015;34(30):3957-3967.

41. Kim SH, Kim MO, Cho YY, et al. ERK1 phosphorylates Nanog to regulate protein stability and stem cell self-renewal. Stem Cell Res. 2014;13(1):1-11.

42. Kwon SK, Lee DH, Kim SY, et al. Ubiquitin-specific protease 21 regulating the K48-linked polyubiquitination of NANOG. Biochemical and Biophysical Research Communications. 2017;482(4):1443-1448.

43. Lim KH, Kim SR, Ramakrishna S, et al. Critical lysine residues of Klf4 required for protein stabilization and degradation. Biochem Biophys Res Commun. 2014;443(4):1206-1210.

44. Vierbuchen T, Ostermeier A, Pang ZP, et al. Direct conversion of fibroblasts to functional neurons by defined factors. Nature. 2010;463:10351041.

45. Han JK, Chang SH, Cho HJ, et al. Direct conversion of adult skin fibroblasts to endothelial cells by defined factors. Circulation. 2014;130(14):1168-1178.

46. Caiazzo M, Giannelli S, Valente P, et al. Direct conversion of fibroblasts into functional astrocytes by defined transcription factors. Stem Cell Reports. 2015;4(1):25-36.

47. Sekiya S, Suzuki A. Direct conversion of mouse fibroblasts to hepatocyte-like cells by defined factors. Nature. 2011;475(7356):390-393.

48. Haq S, Ramakrishna S. Deubiquitylation of Deubiquitylases. Open Biology. 2017;7(6):170016. 